\title{
Erratum to: Generalized outcome-based strategy classification: Comparing deterministic and probabilistic choice models
}

\author{
Benjamin E. Hilbig • Morten Moshagen
}

Published online: 12 September 2014

(C) Psychonomic Society, Inc. 2014

Erratum to: Journal of Psychonomic Bulletin \& Review DOI 10.3758/s13423-014-0643-0

In the Appendix of the article "Generalized outcome-based strategy classification: Comparing deterministic and probabilistic choice models" the equations for the EQW and TTB models were incorrect. The correct model equations are

$$
\begin{aligned}
& \text { EQW (implement with } e_{1}=e_{2} \text { and } e_{3}=.50 \text { ): } \\
& \mathrm{p}(\text { "B" } \mid \text { Item type } 1)=e_{1} \\
& \mathrm{p}(\text { "A" } \text { Item type } 1)=\left(1-e_{1}\right) \\
& \mathrm{p}\left(\text { "A" } \text { Item type 2) }=e_{2}\right. \\
& \mathrm{p}(\text { "B" Item type } 2)=\left(1-e_{2}\right) \\
& \mathrm{p}(\text { "B" Item type } 3)=e_{3} \\
& \mathrm{p}(\text { "A" } \mid \text { Item type } 3)=\left(1-e_{3}\right)
\end{aligned}
$$

TTB (implement with $e_{1}=e_{2}=e_{3}$ ):

$$
\begin{aligned}
& \mathrm{p}(\text { "B" } \mid \text { Item type } 1)=e_{1} \\
& \mathrm{p}(\text { "A" Item type } 1)=\left(1-e_{1}\right) \\
& \mathrm{p}\left(\text { "B" Item type 2) }=e_{2}\right. \\
& \mathrm{p}(\text { "A" Item type } 2)=\left(1-e_{2}\right) \\
& \mathrm{p}(\text { "B" Item type } 3)=e_{3} \\
& \mathrm{p}(\text { "A" Item type } 3)=\left(1-e_{3}\right)
\end{aligned}
$$

The online version of the original article can be found at http:// dx.doi.org/10.3758/s13423-014-0643-0.

B. E. Hilbig $(\bowtie) \cdot$ M. Moshagen

Department of Psychology, School of Social Sciences, University of Mannheim, Schloss Ehrenhof Ost, 68131 Mannheim, Germany e-mail: hilbig@psychologie.uni-mannheim.de 